\title{
Propiedades psicométricas de la Escala de Actitudes hacia la Autoridad Institucional en adolescentes (AAI-A)
}

\author{
${ }^{1}$ Universidad de Valencia \\ ${ }^{2}$ Universidad Miguel Hernández (Elche) \\ ${ }^{3}$ Universidad Pablo de Olavide (Sevilla)
}

María J. Cava ${ }^{1 *}$, Estefanía Estévez ${ }^{2}$, Sofía Buelga ${ }^{1}$, Gonzalo Musitu ${ }^{3}$

\begin{abstract}
Resumen: Las actitudes de los adolescentes hacia la autoridad institucional se relacionan con su implicación en comportamientos transgresores y con conductas de riesgo tales como el consumo de sustancias o las conductas predelictivas. Las actitudes que mantienen hacia profesores, policía, escuela y leyes constituyen, por tanto, una variable relevante en el estudio del ajuste psicosocial de los adolescentes. El objetivo de este trabajo es el de aportar un instrumento fiable para la medición de estas actitudes, analizando las propiedades psicométricas de la Escala de Actitudes hacia la Autoridad Institucional en adolescentes (AAI-A). La estructura factorial de esta escala es analizada mediante análisis factorial exploratorio, análisis confirmatorio y análisis multigrupo utilizando dos muestras independientes de adolescentes escolarizados (1795 españoles y 1494 mexicanos). Los resultados obtenidos muestran una estructura compuesta por dos factores, Actitud positiva hacia la autoridad institucional y Actitud positiva hacia la transgresión de las normas. La escala muestra además adecuados coeficientes de fiabilidad y correlaciones significativas con las conductas agresivas de los adolescentes y con su percepción del profesor en el contexto escolar.

Palabras clave: Adolescentes; actitudes hacia la autoridad; escala; propiedades psicométricas.
\end{abstract}

\section{Introducción}

La actitud del adolescente hacia la autoridad institucional ha demostrado ser una variable relevante en la explicación de su implicación en comportamientos transgresores y en conductas de riesgo tales como el consumo de sustancias o las conductas predelictivas (Emler y Reicher, 2005; Estévez y Emler, 2009). Aunque la percepción de la autoridad parental y la calidad de las relaciones entre padres e hijos tienen una indudable influencia en el ajuste psicosocial del adolescente (Galicia, Sánchez y Robles, 2009; Izabe y Jaureguizar, 2011; Ruiz-Juan y Ruiz-Risueño, 2011), sus actitudes hacia la autoridad formalmente establecida y hacia las normas vinculadas a estas fuentes de autoridad constituyen un aspecto clave en su implicación en conductas transgresoras (Estévez y Emler, 2009; Molpeceres, Llinares y Bernad, 1999; Rigby y Black, 1993; Pons, Molpeceres y Lucas, 2000).

En diversos estudios se han relacionado las conductas violentas de los adolescentes con sus actitudes negativas hacia autoridades e instituciones, tales como la policía, la ley, los profesores o la escuela (Adair, Dixon, Moore y Sutherland, 2000; Emler y Reicher, 1995; Musitu, Estévez y Emler, 2007). Por el contrario, unas actitudes positivas hacia estas fuentes de autoridad se han relacionado con un adecuado ajuste psicosocial del adolescente (Moncher y Miller, 1999). Específicamente dentro del contexto escolar, las actitudes

* Dirección para correspondencia [Correspondence address]: María Jesús Cava. Departamento de Psicología Social. Facultad de Psicología. Universidad de Valencia. Avda. Blasco Ibáñez, 21. 46010. Valencia. E-mail: Maria.J.Cava@uv.es

\begin{abstract}
Title: Psychometric properties of the Attitudes to Institutional Authority in adolescence Scale (AAI-A).

Abstract: Adolescent attitudes to institutional authority are related to their implication in deviant and risk behaviors such as substance abuse and predelinquency. Attitudes towards teachers, the police, the school and laws constitute, therefore, a relevant variable in the study of psychosocial adjustment in adolescence. The objective of the present work is to contribute a reliable instrument for the measurement of these attitudes, and to analyze the psychometric properties of the Attitudes to Institutional Authority in Adolescence Scale (AAI-A). Factor structure of this scale is examined by means of exploratory factor analyses, confirmatory factor analyses, and multigroup analyses, in two independent samples of adolescent students (1795 Spanish and 1494 Mexican). Results obtained show a bifactorial structure, being factors referred to Positive attitude to institutional authority and Positive attitude towards transgression of rules. This scale also shows adequate reliability coefficients and significant correlations with adolescent aggressive behavior and their perception of teachers in the school context.

Key words: Adolescents; attitudes to authority; scale; psychometric properties.
\end{abstract}

negativas hacia la autoridad formal representada por el profesor se han relacionado con conductas violentas dirigidas tanto a profesores como a compañeros (Cava, Musitu y Murgui, 2006; Estévez, Jiménez y Moreno, 2011); siendo actualmente cada vez mayor el interés existente hacia esta variable en los estudios sobre violencia escolar (Estévez et al., 2011).

En sus estudios sobre las actitudes hacia las figuras de autoridad formal, Emler y Reicher $(1995,2005)$ han constatado la importante conexión existente entre las actitudes hacia el profesorado, como fuente de autoridad en el contexto escolar, y las actitudes hacia otras fuentes de autoridad de tipo institucional, tales como la policía. Igualmente, estos investigadores han vinculado las actitudes positivas hacia la transgresión de normas escolares con las actitudes positivas hacia la transgresión de otras normas sociales (Emler, 1993; Emler, Ohana y Dickinson, 1990; Emler, Ohana y Moscovici, 1987; Estévez y Emler, 2009). Para estos investigadores, la experiencia que el adolescente tiene con el profesor constituye con frecuencia su primer contacto con una figura de autoridad formalmente establecida, y el modo en que se desarrolle esta experiencia y cómo el alumno lo perciba en su rol de figura de autoridad influirá en su mayor o menor cumplimiento de las normas escolares, y también en sus actitudes hacia las normas legales y hacia la policía (Emler et al., 1990; Emler y Reicher, 1995, 2005). En sus trabajos han desarrollado diversas escalas para medir las actitudes del adolescente hacia la autoridad formal, en los que han constatado las similitudes existentes entre las actitudes hacia las diferentes figuras de autoridad de tipo formal o institucional 
(Emler y Reicher, 2005). En nuestro contexto nacional, se han realizado algunos estudios, aunque muy escasos, que han permitido constatar también la relación existente entre estas actitudes y determinados comportamientos problemáticos durante la adolescencia (Cava et al., 2006; Cava, Murgui y Musitu, 2008; Moreno, Estévez, Murgui y Musitu, 2009). Sin embargo, y a pesar de la importancia que estas actitudes tienen para analizar el ajuste psicosocial del adolescente, no existe ninguna escala en lengua española que permita realizar una adecuada medición de las mismas.

Aunque algunas escalas de clima escolar y de violencia escolar, disponibles en español, incluyen alguna dimensión relativa a la relación profesor-alumno (Álvarez, ÁlvarezGarcía, González-Castro, Nuñez y González-Pienda, 2006; Alvárez-García, Nuñez, Rodríguez, Alvárez y Dobarro, 2011; Fernández-Ballesteros y Sierra, 1989; Trianes, Blanca, de la Morena, Infante y Raya, 2006), en estas escalas no se evalúan de un modo específico las actitudes hacia el profesor como figura de autoridad institucional ni hacia las normas escolares. Las escalas de actitudes hacia la autoridad institucional desarrolladas por investigadores como Emler (Emler y Reicher, 1995, 2005) o como Palmonari (Gouveia-Pereira, Vala, Palmonari y Rubini, 2003; Palmonari, Rubini y Casoni, 1999; Rubini y Palmonari, 1995, 1998) incluyen ítems relativos a la percepción que el adolescente tiene sobre las normas escolares, la legitimidad del profesor como fuente de autoridad y la imparcialidad de éste en el cumplimiento de las normas. Estas escalas incluyen además ítems referidos a la percepción que el adolescente tiene de otras figuras de autoridad y normas sociales externas al contexto escolar, tales como la policía o las leyes.

En este sentido, y a modo de ejemplo, la escala desarrollada por Gouveia-Pereira et al. (2003) integra diferentes subescalas en las que se evalúa la percepción de justicia en el contexto escolar que tiene el adolescente, con ítems tales como "Las calificaciones que me dan mis profesores son, en general, justas" o "Mis profesores siempre me tratan del mismo modo que a otros estudiantes"; la evaluación que el adolescente realiza de sus experiencias escolares, incluyendo su percepción sobre las normas escolares (p.e. "Las reglas están para el beneficio de los profesores"); y su evaluación de la autoridad, tanto de la autoridad que representan los profesores como de la policía y las leyes (p.e. "Las leyes defienden los derechos de todos los ciudadanos"). Se trata, por tanto, de escalas que no se centran solo en la percepción que el adolescente tiene del profesor y de su labor docente, sino que incluyen también su percepción de las normas escolares, de la figura de autoridad que el profesor representa y de otras normas sociales y figuras de autoridad institucional externas al contexto escolar. Disponer de una escala en español que nos permita medir estas actitudes en los adolescentes, y cuyas propiedades psicométricas hayan sido adecuadamente analizadas, puede permitir una ampliación de las investigaciones sobre las conductas transgresoras de los adolescentes, y contribuir significativamente al desarrollo y evaluación de programas de intervención dirigidos a la prevención de conductas de riesgo en adolescentes.
Teniendo en cuenta la importancia de esta variable, y la carencia de un instrumento adecuado para su medición, en el presente estudio se planteó como objetivo elaborar una escala de actitudes hacia la autoridad institucional para adolescentes y analizar su estructura factorial, fiabilidad y validez. Para el desarrollo de esta escala se realizó previamente tanto una revisión teórica de los estudios sobre actitudes hacia la autoridad como también de las principales escalas elaboradas para su medición (Emler, 1995, 2005; Gouveia-Pereira et al., 2003). Para el análisis de sus propiedades psicométricas y estructura factorial, se realizaron análisis factoriales exploratorios, análisis confirmatorios y análisis multigrupo utilizando dos muestras independientes de adolescentes (españoles y mexicanos). También, se analizó la consistencia interna de la escala y su validez convergente mediante el análisis de sus relaciones con dos variables con las que se encuentra teóricamente relacionada, las conductas agresivas del adolescente y su percepción del profesor. Mediante este estudio, se pretende aportar un instrumento fiable para la medición de la actitud de los adolescentes hacia la autoridad institucional y hacia las normas socialmente establecidas.

\section{Método}

\section{Participantes}

En la realización de este estudio se utilizaron dos muestras independientes de adolescentes escolarizados. La primera muestra estaba formada por 1795 adolescentes españoles de ambos sexos (48\% mujeres) y con edades comprendidas entre los 11 y 18 años $(M=14.20, D T=1.68)$. Estos adolescentes cursaban sus estudios en 9 centros educativos, públicos y concertados, de la Comunidad Autónoma de Andalucía, distribuidos en $1^{\circ}, 2^{\circ}, 3^{\circ}$ y $4^{\circ}$ de Secundaria; y $1^{\circ}$ y $2^{\circ}$ de Bachiller. La segunda muestra estaba compuesta por 1494 adolescentes mexicanos que cursaban sus estudios en 6 Centros educativos, públicos y concertados, del Estado de Sinaloa (México), de ambos sexos (55\% mujeres), con edades comprendidas entre los 12 y 19 años $(M=14.82$ años, $D T=$ 1.75) y distribuidos en los cursos de $1^{\circ}, 2^{\circ}$ y $3^{\circ}$ de Secundaria; y $1^{\circ}, 2^{\circ}$ y $3^{\circ}$ de Preparatoria.

\section{Instrumentos}

La escala de Actitudes hacia la Autoridad Institucional para Adolescentes (AAI-A) fue elaborada a partir de la escala previa de Actitudes hacia la Autoridad de Reicher y Emler (1995) y de las escalas de Percepción de Justicia en el Contexto Escolar, Evaluación de la Experiencia Escolar y Evaluación de la Autoridad de Gouveia-Pereira et al. (2003). Algunas adaptaciones previas de la escala de Reicher y Emler (1995), realizadas en estudios sobre conductas de riesgo en adolescentes (Cava et al., 2006; Moreno et al., 2009), fueron también consideradas para la selección definitiva de los ítems. La escala elaborada consta de 10 ítems relativos a la actitud de los adolescentes hacia el profesorado y hacia la 
policía como figuras de autoridad (p.e. "Los profesores son justos a la hora de evaluar", "La policía está para hacer una sociedad más justa para todos"), y hacia las reglas escolares y la ley como sistemas normativos (p.e. "Si una regla escolar no te gusta, lo mejor es saltártela", "Es normal saltarse la ley si no se causa daño a nadie"). A estos ítems se responde mediante una escala tipo Likert con cuatro opciones de respuesta $(1=$ nada de acuerdo; $2=$ algo de acuerdo; $3=$ bastante de acuerdo; $4=$ totalmente de acuerdo).

Para analizar su validez convergente, se utilizaron otras dos escalas: la de Conducta Violenta en la Escuela de Little, Henrich, Jones y Hawley (2003; adaptación española de Estévez, Murgui, Musitu y Moreno, 2008) y la de Clima Escolar de Moos y Trickett (1973; adaptación española de Fernández-Ballesteros y Sierra, 1989). La Escala de Conducta Violenta en la Escuela de Little et al. (2003) consta de 25 ítems que evalúan, con un rango de respuesta de 1 a 4 (nunca, pocas veces, muchas veces y siempre), diversos tipos de conducta violenta en el contexto escolar. En este estudio se consideraron las tres subescalas que miden agresión manifiesta, en su forma pura (p.e., "Soy una persona que se pelea con los demás"), reactiva (p.e. "Cuando alguien me hace daño o me biere, le pego") e instrumental (p.e. "Amenazo a otros para conseguir lo que quiero"). Estas tres subescalas presentaron adecuados coeficientes de fiabilidad. En la muestra española la fiabilidad (alfa de Cronbach) fue de .68 para la subescala de agresión manifiesta pura, .77 para la subescala de agresión manifiesta reactiva y .81 para la subescala de agresión manifiesta instrumental. En la muestra mexicana los coeficientes obtenidos para estas tres subescalas fueron, respectivamente, .65, .79 y .80

La Escala de Clima Escolar de Moos y Trickett (1973) mide la percepción que los alumnos tienen del clima del aula, especialmente de la relación profesor-alumno, las relaciones entre alumnos y la estructura organizativa. En este estudio se consideró únicamente la subescala de Ayuda del Profesor, relativa a la percepción que el alumno tiene sobre el grado de ayuda, preocupación y amistad que el profesor muestra hacia los alumnos (p.e. "Este profesor dedica muy poco tiempo a hablar con los alumnos"). Está compuesta por 10 ítems de verdaderofalso, y su coeficiente de fiabilidad (alpha de Cronbach) es de .67 para la muestra española y de .66 para la mexicana.

\section{Procedimiento}

Previamente a la investigación, se envió una carta a los centros educativos seleccionados explicándoles los objetivos del proyecto y solicitándoles su colaboración. Se siguió este mismo procedimiento en ambas muestras. Posteriormente, se contactó telefónicamente con la dirección de los centros y se concertó una entrevista para explicar más detenidamente el objetivo de la investigación. En esta reunión se entregaron también a los centros los consentimientos informados para los padres junto con una carta explicativa. Tras la obtención de los permisos correspondientes, se realizó la aplicación del instrumento. Esta aplicación se llevó a cabo por investigado- res expertos y entrenados, en las aulas habituales y durante un período regular de clase. Los adolescentes fueron informados de que su participación en la investigación era voluntaria y anónima.

\section{Análisis de datos}

En primer lugar, se realizó un Análisis Factorial Exploratorio con la muestra española utilizando el paquete estadístico SPSS (versión 17), y se calculó la fiabilidad de los factores. Posteriormente, se realizaron Análisis Confirmatorios utilizando el programa EQS 6.1, y procediendo del siguiente modo: 1) se contrastó en la muestra mexicana si la estructura factorial obtenida previamente representaba razonablemente los datos (validación cruzada); 2) a través de análisis multigrupo y análisis de estructuras de covarianza que incluyen también la media (Latent Mean Structures) se estimó la invarianza factorial de la estructura encontrada para la muestra española (por país y por género). Todos los resultados del ajuste de los modelos se basaron en la solución robusta, debido a la desviación de la normalidad observada en los datos. Esta solución robusta aplica una corrección a la desviación de la multinormalidad de los datos para estimar un $\chi^{2}$ ajustado $\left(\mathrm{S}-\mathrm{B} \chi^{2}\right)$, errores típicos robustos e índices de ajuste robustos. Finalmente, para analizar la validez convergente de la escala, se realizaron, mediante el cálculo del coeficiente de Pearson, análisis de correlación entre las dimensiones de la escala y las variables teóricamente relacionadas.

\section{Resultados}

\section{Análisis Factorial Exploratorio y Fiabilidad}

Los resultados de la prueba de Kaiser-Meyer-Olkin (.82) y de la prueba de Barlett $\left(\chi^{2}=3740.31, g l=45, p<.001\right)$ realizados con la muestra española fueron satisfactorios, por lo que se realizó el Análisis Factorial Exploratorio. El método utilizado fue el de Factorización de Ejes Principales. Se utilizó la rotación Oblimin, puesto que se consideraba probable que existieran correlaciones significativas entre los factores. Una vez obtenidos los resultados del análisis factorial, la selección del número de factores se realizó teniendo en cuenta varios criterios. En primer lugar, se observó que dos factores mostraban autovalores iniciales superiores a 1 (regla de Kaiser). El gráfico de sedimentación también mostró una caída importante de la pendiente a partir del segundo factor. Por último, se utilizó también como criterio el análisis paralelo de Horn (Hayton, Allen y Scarpello, 2004). Este método compara los autovalores de los factores obtenidos con los autovalores que se obtendrían mediante la factorización de una muestra de datos al azar de igual tamaño y mismo número de variables. En concreto, se comparan los autovalores obtenidos con los datos reales con la media (o el percentil 95) de los autovalores obtenidos en la factorización de 50 muestras de datos al azar de similares características. Si los autovalores de los factores reales son superiores a sus paralelos obtenidos con datos al azar, se considera que ese fac- 
tor debe mantenerse. En la factorización de nuestros datos, el primer factor resultante mostró un autovalor inicial de 3.385 y el segundo factor un autovalor inicial de 1.602. La media de los factores paralelos (obtenidos mediante muestras al azar) fue para los factores 1 y 2 , respectivamente, de 1.1191 y 1.0845, y su percentil 95 fue, respectivamente, de 1.1625 y 1.1109. En ambos casos, los autovalores de los datos reales superaron a los autovalores de sus factores paralelos, por lo que se decidió mantener ambos factores.

Los dos factores obtenidos explican en conjunto el $39.06 \%$ de la varianza (ver Tabla 1). Analizando el contenido de los ítems que se distribuyen en cada uno de ellos, se observa una estructura coherente desde el punto de vista teórico. El primer factor, al que denominamos Actitud Positiva hacia la Autoridad, incluye ítems que reflejan una actitud positiva del adolescente hacia el profesorado y hacia la policía como figuras de autoridad. Las actitudes hacia ambos tipos de autoridad quedan incluidas en un único factor que explica el $28.13 \%$ de la varianza. El segundo factor, al que denominamos, Actitud Positiva hacia la Transgresión, incluye ítems que hacen referencia a una actitud positiva del adolescente hacia la transgresión de la ley y las normas escolares. Este segundo factor explica el $10.93 \%$ de la varianza. Todos los ítems muestran saturaciones superiores a .40 en un único factor, a excepción del ítem 3. Además, si observamos los índices de homogeneidad de los ítems (Tabla 1) se aprecia también cómo el ítem 3 muestra un valor inferior a .30, lo que aconsejaría su eliminación de la escala. Este ítem queda, por tanto, excluido de la escala y no será considerado en los posteriores análisis. Los coeficientes de consistencia interna ( $\alpha$ de Cronbach) de los dos factores obtenidos, fueron, respectivamente, .75 y .74 . Ambos factores muestran una correlación de -.43.

Tabla 1. Medias, desviaciones típicas (DT) e índices de homogeneidad de los ítems y Análisis Factorial Exploratorio (muestra española).

\begin{tabular}{|c|c|c|c|c|}
\hline \multirow[t]{4}{*}{ Media } & \multirow[t]{4}{*}{$D T$} & \multirow[t]{4}{*}{$\begin{array}{c}\text { Indice de } \\
\text { Homogeneidad } \\
\end{array}$} & \multicolumn{2}{|c|}{ Análisis Factorial Exploratorio } \\
\hline & & & F1 & F2 \\
\hline & & & Actitud positiva & Actitud positiva \\
\hline & & & Autoridad & Transgresión \\
\hline 2.63 & .83 & .563 & .67 & -.26 \\
\hline 3.22 & .85 & .451 & .54 & -.33 \\
\hline 3.52 & .71 & .124 & .29 & -.14 \\
\hline 1.78 & .86 & .440 & -.34 & .53 \\
\hline 2.29 & 1.02 & .517 & .60 & -.21 \\
\hline 2.79 & 1.01 & .452 & .53 & -.31 \\
\hline 2.56 & .81 & .623 & .77 & -.25 \\
\hline 1.77 & .91 & .503 & -.21 & .64 \\
\hline 1.64 & .89 & .615 & -.29 & .83 \\
\hline 1.43 & .74 & .528 & -.30 & .62 \\
\hline
\end{tabular}

1. Los profesores son justos a la hora de evaluar

2. La policía está para hacer una sociedad mejor para todos

3. Ir bien en la escuela ayuda a tener éxito en la vida

4. Es normal saltarse la ley si no se causa daño a nadie

5. Los profesores tratan igual a todos los estudiantes

6. Si viese a alguien robar se lo diría a la policía

7. Estoy de acuerdo con lo que hacen y dicen la mayoría de los profesores

8. Es normal desobedecer a los profesores si no hay castigos

9. Da igual saltarse las reglas escolares si después no hay castigos

10. Si una regla escolar no te gusta, lo mejor es saltártela

\section{Análisis Confirmatorios}

A continuación, teniendo en cuenta los factores obtenidos, se realizaron los Análisis Confirmatorios. En concreto, se examinó mediante este análisis un modelo de dos factores correlacionados para los 9 ítems de la escala con la muestra mexicana. Para este análisis se utilizó una segunda muestra con el objeto de realizar una validación cruzada de la estructura factorial obtenida (Worthington y Whitttaker, 2006).

Se utilizó el método de Estimación de Máxima Verosimilitud Robusta (Robust Maximum Likelihood Estimation) para todos los análisis, debido a la falta de normalidad multivariada de los datos (coeficiente Mardia= 12.56). Si observamos en la Tabla 2 los coeficientes de asimetría y curtosis de los ítems que integran la escala, apreciamos una ausencia de normalidad moderada. Así, observamos que la mayoría de los coeficientes de asimetría se sitúan entre +1 y -1 , y únicamente el ítem 10 presenta un valor ligeramente superior (1.0274). En cuanto a los coeficientes de curtosis, se sitúan también la mayoría con valores entre +1 y -1 . Aunque el método de Máxima Verosimilitud asume el supuesto de la normalidad multivariada, resulta razonablemente robusto a un incumplimiento moderado (Muthén y Kaplán, 1985), y en todo caso, el posible sesgo en la estimación se produciría en el sentido de aportar unos índices de ajuste peores a los reales (García, Musitu, Riquelme y Riquelme, 2011). La Chicuadrado escalada Satorra-Bentler $\left(\mathrm{SB} \chi^{2}\right)$, utilizada en estos análisis, ha sido recomendada en casos en que hay una ausencia moderada de normalidad en la distribución de los datos (Curran, West y Finch, 1996).

Por otra parte, y teniendo en cuenta las actuales recomendaciones sobre la conveniencia de no utilizar una única medida de ajuste del modelo, sino varios índices de diferentes tipos de medidas (Bentler, 2007; Goffin, 2007; Markland, 2007; Miles y Shevlin, 2007; Mulaik, 2007), hemos considerado, además de la Chi-cuadrado (que además, puede verse afectada por el tamaño de la muestra), los siguientes índices de ajuste: el índice de ajuste comparativo robusto (CFI robusto), el índice de ajuste no normado de Bentler-Bonett (NNFI) o índice de Tucker-Lewis (TLI) y el error de aproximación cuadrático medio (RMSEA). Aunque no deben considerarse como puntos de corte fijos (Markland, 2007), habitualmente son considerados como un buen ajuste del modelo índices del CFI y del NNFI superiores a .95, y 
aceptables si superan el .90. En el caso del RMSEA se considera un buen ajuste valores inferiores a 0.50 , y un ajuste aceptable valores situados entre .50 y .80 . En nuestro modelo, estos índices mostraron los siguientes valores: $\mathrm{SB} \chi^{2}=$
$81.60, g l=25, p<.001, \mathrm{CFI}=.967, \mathrm{NNFI}=.953 ; \mathrm{RMSEA}$ $=.046(.037,-.054)$. Estos valores, tomados en conjunto, indican un buen ajuste del modelo y, por tanto, confirman la estructura factorial previamente obtenida.

Tabla 2. Medias, desviaciones típicas (DT), asimetría y curtosis de los ítems y Análisis Factorial Confirmatorio (muestra mexicana).

\begin{tabular}{|c|c|c|c|c|c|c|}
\hline & \multirow[t]{2}{*}{ Media } & \multirow[t]{2}{*}{$D T$} & \multirow[t]{2}{*}{ Asimetría } & \multirow[t]{2}{*}{ Curtosis } & \multicolumn{2}{|c|}{$\begin{array}{l}\text { Análisis Factorial Confirmatorio: } \\
\text { Solución Estandarizada }\end{array}$} \\
\hline & & & & & $\begin{array}{c}\text { F1 } \\
\text { Actitud positiva } \\
\text { Autoridad }\end{array}$ & $\begin{array}{c}\text { F2 } \\
\text { Actitud positiva } \\
\text { Transgresión }\end{array}$ \\
\hline 1. Los profesores son justos a la hora de evaluar & 2.32 & .94 & .4699 & -.6691 & .59 & \\
\hline 2. La policía está para hacer una sociedad mejor para todos & 2.63 & 1.07 & -.0680 & -.2878 & .44 & \\
\hline 4. Es normal saltarse la ley si no se causa daño a nadie & 1.87 & .94 & .8539 & -.2171 & & .44 \\
\hline 5. Los profesores tratan igual a todos los estudiantes & 1.99 & 1.01 & .7326 & .6118 & .68 & \\
\hline 6. Si viese a alguien robar se lo diría a la policía & 2.78 & 1.02 & -.2636 & -1.2252 & .37 & \\
\hline $\begin{array}{l}\text { 7. Estoy de acuerdo con lo que hacen y dicen la mayoría de } \\
\text { los profesores }\end{array}$ & 2.26 & .87 & .3818 & -.1984 & .67 & \\
\hline 8. Es normal desobedecer a los profesores si no hay castigos & 2.00 & 1.00 & .6868 & -.6215 & & .60 \\
\hline $\begin{array}{l}\text { 9. Da igual saltarse las reglas escolares si después no hay cas- } \\
\text { tigos }\end{array}$ & 1.90 & .99 & .8151 & -.4613 & & .79 \\
\hline 10. Si una regla escolar no te gusta, lo mejor es saltártela & 1.80 & .98 & 1.0274 & .0871 & & .61 \\
\hline
\end{tabular}

Todos los coeficientes del Análisis Confirmatorio son significativos $(p<.001)$.

\section{Análisis Multigrupo}

Seguidamente, se evalúo la invarianza factorial del cuestionario de Actitudes hacia la Autoridad Institucional. Para ello, se hipotetizó que el número de factores, las saturaciones de los ítems, y las correlaciones entre los dos factores eran invariantes en las muestras de España y México. Asimismo, se planteó también como hipótesis esta invarianza factorial entre los chicos y chicas de cada una de las dos muestras. Estas hipótesis se evaluaron mediante el análisis multigrupo, el cual permite establecer igualdades adicionales en dos o más grupos y obtener la probabilidad de que esas igualdades existan en la población. En concreto, se evaluaron tres modelos multigrupo para realizar las siguientes comparaciones: España-México, Chicas-Chicos en España, y Chicas-Chicos en México.

Para la evaluación de los modelos multigrupo se estimaron diferentes modelos anidados y se compararon a través del test de la diferencia en $\chi^{2}$. Los modelos anidados fueron los siguientes: a) un primer modelo multigrupo que no impone ninguna igualdad entre las saturaciones y las correlaciones entre los factores (modelo sin restricciones); y, b) un segundo modelo que impone la igualdad entre todas las saturaciones y las correlaciones entre los factores (modelo con restricciones) en los grupos. La diferencia en $\chi^{2}$ de estos dos modelos se distribuye también como $\chi^{2}$ con grados de libertad igual a la diferencia entre los grados de libertad de ambos modelos. Si se acepta la hipótesis nula (que los modelos son equivalentes estadísticamente) ello significa que el modelo con mayor número de grados de libertad (el modelo más restringido) es preferible. Finalmente, si es el caso, se liberan aquellas restricciones (igualdades entre grupos) que en el modelo más restringido llevan a una pérdida de ajuste y se analizan esos parámetros individualmente para cada grupo. Un resumen de los índices de ajuste multigrupo y el test de la diferencia en $\chi^{2}$ se presenta en la Tabla 3 .

Tabla 3. Modelo General para España y México.

\begin{tabular}{|c|c|c|c|c|c|}
\hline Modelo & $\mathrm{S}-\mathrm{B} \times 2$ & $g l$ & $p$ & CFI robusto & RMSEA robusto \\
\hline \multicolumn{6}{|l|}{ Invarianza Factorial España-México } \\
\hline (a) Modelo sin restricciones & 211.11 & 50 & $<.001$ & 0.97 & $.04(.04-.05)$ \\
\hline (b) Modelo con restricciones & 234.23 & 57 & $<.001$ & 0.97 & $.04(.04-.05)$ \\
\hline Modelo b - Modelo $\mathrm{a}^{1}$ & 23.12 & 7 & .001 & & \\
\hline (c) Modelo con 2 restricciones liberadas & 218.56 & 55 & $<.001$ & 0.97 & $.04(.04-.05)$ \\
\hline Modelo c - Modelo a & 7.45 & 5 & .19 & & \\
\hline \multicolumn{6}{|c|}{ Invarianza Factorial España (Chicos y Chicas) } \\
\hline (a) Modelo sin restricciones & 127.57 & 50 & $<.001$ & 0.97 & $.04(.03-.05)$ \\
\hline (b) Modelo con restricciones & 139.60 & 57 & $<.001$ & 0.97 & $.04(.03-.05)$ \\
\hline Modelo b - Modelo a & 12.04 & 7 & .09 & & \\
\hline \multicolumn{6}{|c|}{ Invarianza Factorial México (Chicos y Chicas) } \\
\hline (a) Modelo sin restricciones & 93.30 & 50 & $<.001$ & 0.98 & $.03(.02-.04)$ \\
\hline (b) Modelo con restricciones & 104.14 & 57 & $<.001$ & 0.97 & $.03(.02-.04)$ \\
\hline Modelo b - Modelo a & 10.84 & 7 & .15 & & \\
\hline
\end{tabular}

${ }^{1}$ Las comparaciones de modelos basadas en el $\chi 2$ de Satorra-Bentler se realizan siguiendo el procedimiento descrito en Crawford y Henry (2003). 
Con respecto a la comparación simultánea de la estructura factorial entre la muestra española y la mexicana, se observa que no se puede mantener la igualdad entre los modelos sin ninguna restricción (Modelo a) y con todas las restricciones (Modelo b) $\left(\Delta \chi^{2}=23.12, \Delta \mathrm{gl}=7, p<.001\right)$ por lo que es necesario re-estimar el modelo liberando aquellas igualdades que llevan a una falta de ajuste al modelo. Estas restricciones estadísticamente significativas se identifican en el programa EQS a partir del Multiplicador de Lagrange. En el caso de la comparación España-México, dos igualdades entre factores e ítems son distintas en los grupos y por tanto habría que liberar esas igualdades (la asociación entre el factor Actitud Positiva hacia la Autoridad y el ítem 6, para España resultó $\beta=.496, p<.001$, mientras que en el caso de México fue $\beta=.449, p<.001$; y la asociación entre el factor Actitud Positiva hacia la Transgresión de normas sociales y el ítem 10, para España resultó $\beta=0.736, p<.001$, mientras que en el caso de México fue $\beta=0.638, p<.001)$. Este nuevo modelo (c) es estadísticamente equivalente al modelo (a) $\left(\Delta \chi^{2}=7.45, \Delta \mathrm{gl}=5, p=.19\right)$ y por tanto se puede mantener que las igualdades en las saturaciones son idénticas para España y México.

En cuanto a las comparaciones en función del género, siguiendo con la muestra española, se observa en la tabla que el modelo sin restricciones (Modelo a) y el modelo con restricciones (Modelo b) son estadísticamente equivalentes,
$\left(\Delta \chi^{2}=12.04, \Delta \mathrm{gl}=7, p=.09\right)$, por lo que se puede mantener que la estructura factorial es idéntica para chicos y chicas, incluidas las correlaciones entre los factores. En el caso de la muestra mexicana, el modelo sin restricciones (Modelo a) y el modelo con restricciones (Modelo b) son estadísticamente equivalentes $\left(\Delta \chi^{2}=10.84, \Delta \mathrm{gl}=7, p=.15\right)$. Por tanto, también se puede mantener en esta muestra que la estructura factorial es idéntica para chicos y chicas, incluidas las correlaciones entre los factores.

\section{Análisis de Correlación}

Por último, se analizó la validez convergente de la escala considerando las correlaciones entre los dos factores que la componen y otros constructos con los que teóricamente se encuentran relacionados. En concreto, se analizaron las correlaciones entre los dos factores de la Escala de Actitudes hacia la Autoridad Institucional (AAI-A) y las subescalas de Ayuda del profesor (Escala de Clima Escolar de Moos y Trickett, 1973), Agresión manifiesta pura, Agresión manifiesta reactiva y Agresión manifiesta instrumental (Escala de Conducta Violenta en la Escuela de Little et al., 2003). Estos análisis se realizaron con la muestra española y con la muestra mexicana (ver Tabla 4).

Tabla 4. Correlaciones y tamaño del efecto (entre paréntesis) entre Ayuda del profesor, Agresión manifiesta y factores de la escala de Actitudes hacia la Autoridad Institucional (AAI-A).

\begin{tabular}{lcccc}
\hline & \multicolumn{2}{c}{ Muestra Española } & \multicolumn{2}{c}{ Muestra Mexicana } \\
\cline { 2 - 5 } & $\begin{array}{c}\text { F1 } \\
\text { Actitud positiva } \\
\text { Autoridad }\end{array}$ & $\begin{array}{c}\text { F2 } \\
\text { Actitud positiva } \\
\text { Transgresión }\end{array}$ & $\begin{array}{c}\text { F1 } \\
\text { Actitud positiva } \\
\text { Autoridad }\end{array}$ & $\begin{array}{c}\text { Actitud positiva } \\
\text { Transgresión }\end{array}$ \\
\hline Ayuda del profesor & $.46(1.0)$ & $-.28(.6)$ & $.34(.7)$ & $-.20(.4)$ \\
Agresión manifiesta pura & $-.30(.6)$ & $.40(.8)$ & $-.17(.3)$ & $.39(.8)$ \\
Agresión manifiesta reactiva & $-.27(.5)$ & $.40(.8)$ & $-.20(.4)$ & $.39(.8)$ \\
Agresión manifiesta instrumental & $-.24(.5)$ & $.38(.8)$ & $-.17(.3)$ & $.38(.8)$ \\
\hline
\end{tabular}

Todas las correlaciones son significativas $(p<.001)$.

Tamaño del efecto $\mathrm{d}>.80$ elevado, $\mathrm{d}>.50$ medio y $\mathrm{d}>.20$ bajo (Cohen, 1988).

En ambas muestras las correlaciones entre todas las variables consideradas fueron estadísticamente significativas $(p$ $<.001)$, el tamaño del efecto mostró valores medios y elevados en la mayoría de las correlaciones (ver Tabla 4), y el signo de estas correlaciones fue el esperado desde un punto de vista teórico. Las correlaciones más elevadas se constatan, en ambas muestras, entre la percepción de ayuda del profesor y la actitud positiva hacia la autoridad institucional $(r=.46 \mathrm{pa}-$ ra la muestra española y $r=.34$ para la muestra mexicana); y entre las distintas formas de agresión y la actitud positiva hacia la transgresión de las normas $(r=.40 ; r=.40 ; r=.38$ para la muestra española y $r=.39 ; r=.39 ; r=.38$ para la muestra mexicana). Estas correlaciones son de signo positivo. Por otra parte, se constatan correlaciones negativas entre la percepción de ayuda del profesor y la actitud positiva hacia la transgresión de las normas $(r=-.28$ para la muestra española y $r=-.20$ para la muestra mexicana), y entre las tres formas de agresión consideradas y la actitud positiva hacia la autoridad institucional $(r=-.30 ; r=-.27 ; r=-.24$ para la muestra española y $r=-.17 ; r=-.20 ; r=-.17$ para la muestra mexicana).

\section{Discusión}

El objetivo de este estudio fue analizar las propiedades psicométricas y la estructura factorial de la escala de Actitudes hacia la Autoridad Institucional en adolescentes (AAI-A). Esta escala se fundamenta en las escalas previas de Emler y Reicher (1995, 2005) y Gouveia-Pereira et al. (2003), y fue desarrollada con la finalidad de suplir la carencia existente en español de un instrumento fiable que permita la adecuada medición de esta variable, estrechamente relacionada con el ajuste psicosocial de los adolescentes (Cava et al., 2006, 2008; Emler y Reicher, 2005; Estévez y Emler, 2009; Esté- 
vez et al., 2011; Moncher y Miller, 1999). Los resultados obtenidos constatan las adecuadas propiedades psicométricas de esta escala.

Los análisis factoriales realizados muestran una estructura compuesta por dos factores, con una alta coherencia desde un punto de vista teórico. El primer factor, denominado Actitud Positiva hacia la Autoridad, hace referencia a la actitud del adolescente hacia el profesorado y la policía como figuras de autoridad institucional formalmente establecidas. Las actitudes hacia ambas figuras de autoridad se integran, por tanto, en un único factor. Este resultado es coincidente con los obtenidos por Emler y Reicher (1995, 2005), Palmonari y Rubini (1998) y Gouveia-Pereira et al. (2003), en los que se constata también la existencia de una actitud general hacia la autoridad formal en la que se incluyen las actitudes del adolescente hacia el profesorado y hacia la policía. El segundo factor, denominado Actitud Positiva hacia la Transgresión, evalúa la percepción que el adolescente tiene de las normas escolares y de la ley, así como su actitud hacia la transgresión de las mismas. Ambos tipos de sistemas normativos quedan también integrados en un único factor, lo que resulta también coincidente con los trabajos de Emler (Emler, 1993; Emler y Reicher, 1995, 2005; Estévez y Emler, 2009) y Palmonari (Palmonari, Rubini y Casoni, 1999; Rubini y Palmonari, 1998). Esta estructura factorial ha sido, además, confirmada en dos muestras independientes de dos países diferentes, y tanto en chicas como en chicos. Los análisis multigrupo realizados confirmaron que la estructura factorial era similar en los distintos grupos considerados.

La escala de Actitudes hacia la Autoridad Institucional en Adolescentes (AAI-A) muestra, además, unos coeficientes de fiabilidad adecuados y correlaciones significativas con las conductas agresivas de los adolescentes y con su percepción de ayuda del profesor. Estos resultados, además de avalar la idoneidad del instrumento, confirman la vinculación establecida en las teorías de Emler y Reicher $(1995,2005)$ entre la relación del adolescente con el profesorado y sus actitudes hacia la autoridad institucional. Estas actitudes parecen ser un elemento clave en el ajuste psicosocial del adolescente, al relacionarse directamente con su implicación en comportamientos transgresores y conductas de riesgo (Cava et al., 2006; 2008; Estévez et al., 2011; Moncher y Miller, 1999), incluida la violencia escolar cuyas consecuencias son altamente negativas (Cava, Buelga, Musitu y Murgui, 2010). Se trata, por tanto, de una variable respecto de la cual resulta de especial relevancia disponer de un instrumento que permita su correcta medición. El instrumento analizado en este estudio presenta unas adecuadas propiedades psicométricas y puede contribuir a ampliar las investigaciones sobre la implicación de los adolescentes en comportamientos problemáticos, tales como la violencia escolar, el consumo de sustancias o las conductas predelictivas. Esta escala puede ser también de utilidad en el desarrollo y evaluación de programas de inter- vención desarrollados en el contexto escolar y dirigidos a potenciar el ajuste psicosocial de los adolescentes.

Por otra parte, la diferenciación que se establece entre ambas dimensiones, actitudes hacia la autoridad y actitudes hacia las normas, resulta también relevante desde un punto de vista teórico, puesto que puede ser de especial utilidad en los estudios sobre estilos de socialización y conductas transgresoras en la adolescencia. La mayoría de los trabajos sobre estilos de socialización parental destacan la relación entre un estilo autorizativo (caracterizado por afecto y severidad) y un adecuado ajuste psicosocial de los hijos (Baumrind, 1971; Darling y Steinberg, 1993). No obstante, estudios recientes han cuestionado que el componente de severidad sea necesario para una adecuada socialización (De la Torre, Casanova, García, Carpio y Cerezo, 2011; García y Gracia, 2009, 2010; Musitu y García, 2004). En este sentido, sería interesante analizar en futuras investigaciones la importancia de estos dos componentes, actitudes hacia la autoridad y actitudes hacia las normas, dentro del contexto familiar.

La escala AAI-A permite, en definitiva, evaluar las actitudes hacia la autoridad institucional en los adolescentes y suplir la carencia existente de un instrumento en español para su adecuada medición. Aunque entre las limitaciones de este trabajo podría incluirse la utilización de autoinformes para evaluar las conductas agresivas de los adolescentes, en estudios previos se ha señalado la viabilidad de este tipo de instrumentos en la medición de la violencia escolar (Crick y Bigbee, 1998). No obstante, sería interesante en futuros estudios continuar analizando la relación entre estas actitudes y las conductas agresivas del adolescente contando también con informes de padres y profesores sobre estas conductas. Igualmente, sería interesante analizar las relaciones entre la actitud del adolescente hacia las figuras de autoridad institucional y su actitud hacia la autoridad paterna, teniendo además en cuenta posibles variaciones en las diferentes etapas de la adolescencia. En futuros trabajos, sería conveniente analizar en mayor medida las relaciones entre las actitudes hacia la autoridad institucional y algunas dimensiones del clima escolar, tales como la relación con los compañeros, las situaciones de exclusión y rechazo social en el aula, las relaciones entre familia y escuela o el rendimiento académico del adolescente. El análisis específico de la influencia de estas actitudes en los distintos tipos de comportamientos de riesgo durante la adolescencia, y el modo en que estas actitudes pueden ser también influidas por el grupo de iguales, son también aspectos que convendría analizar en futuras investigaciones.

Nota de los autores.- Este artículo ha sido elaborado en el marco del proyecto de investigación PSI2008-01535/PSIC "Violencia escolar, victimización y reputación social en la adolescencia”, subvencionado por el Ministerio de Ciencia e Innovación de España. 


\section{Referencias}

Adair, V. A., Dixon, R.S., Moore, D. W. y Sutherland, C. M. (2000). Bullying in New Zealand secondary schools. New Zealand Journal of Educational Studies, 35, 207-221.

Álvarez, L. Álvarez-García, D., González-Castro, P., Nuñez J.C. y GonzálezPienda, J. A. (2006). Evaluación de los comportamientos violentos en los centros educativos. Psicothema, 18(4), 686-695.

Alvárez-García, D., Nuñez, J. C., Rodríguez, C., Alvárez L. y Dobarro, A. (2011). Propiedades psicométricas del Cuestionario de Violencia Escolar- Revisado (CUVE-R). Revista de Psicodidáctica, 16 (1), 59-83.

Baumrind, D. (1971). Current theories of parental authority. Developmental Psychology Monographs, 4(1, part 2), 1-103.

Bentler, P. M. (2007). On tests and indices for evaluating structural models. Personality and Individual Differences, 42, 825-829.

Cava, M. J. Buelga, S., Musitu, G. y Murgui, S. (2010). Violencia escolar entre adolescentes y sus implicaciones en el ajuste psicosocial: un estudio longitudinal. Revista de Psicodidáctica, 15 (1), 21-34.

Cava, M. J., Murgui, S. y Musitu, G. (2008). Diferencias en factores de protección del consumo de sustancias en la adolescencia temprana y media. Psicothema, 20(3), 389-395.

Cava, M. J., Musitu, G. y Murgui, S. (2006). Familia y violencia escolar: el rol mediador de la autoestima y la actitud hacia la autoridad institucional. Psicothema, 18(3), 367-373.

Cohen, J. (1988). Statistical power analysis for the behavioral sciences (2nd ed.). Hillsdale, NJ: Lawrence Earlbaum Associates.

Crawford, J. R. y Henry, J. D. (2003). The Depression Anxiety Stress Scales: Normative data and latent structure in a large non-clinical sample. British Journal of Clinical Psychology, 42, 111-131.

Crick, N. R. y Bigbee, M. A. (1998). Relational and overt forms of peer victimization: A multi-informant approach. Journal of Consulting and Clinical Psychology, 66, 337-347.

Curran, P. J., West, S. G. y Finch, J. F. (1996). The robustness of test statistics to nonnormality and specification error in confirmatory factor analysis. Psichological Methods, 1, 16-29.

Darling, N. y Steinberg, L. (1993). Parenting style as context: An integrative model. Psychological Bulletin, 113, 487-496.

De la Torre, M. J., Casanova, P. F., García, M. C., Carpio, M. V. y Cerezo, M. T. (2011). Parenting styles and stress in students of compulsory secondary education. Behavioral Psychology-Psicologia Conductual, 19, 577-590.

Emler, N. (1993). The young person's relationship to institutional order. En S. Jackson y H. Rodríguez-Tomé (Eds.), Adolescence and its social words (pp. 229-250). Hove: Lawrence Erlbaum Associates.

Emler, N. y Reicher, S. (1995). Adolescence and delinquency. Oxford: Blackwell.

Emler, N. y Reicher, S. (2005). Delinquency: cause or consequence of social exclusion? En D. Abrams, J. Marques y M. Hogg (Eds.), The social psychology of inclusion and exclusion (pp. 211-241). Philadelphia: Psychology Press.

Emler, N., Ohana, J. y Dickinson, J. (1990). Children's representation of social relations. En G. Duveen y B. Lloyd (Eds.), Social representations and the development of knowledge. Cambridge: Cambridge University Press.

Emler, N., Ohana, J. y Mosovici, S. (1987). Children's beliefs about institutional roles: A cross-national study of representations of the teacher's role. British Journal of Educational Psychology, 57, 26-37.

Estévez, E. y Emler, N. (2009). Individual differences in attitude to school and social reputation among peers: Implications for behavioural adjustment in educational settings. En J. E. Larson (Ed.), Educational Psychology (pp. 342-365). Nova.

Estévez, E., Jiménez, T. y Moreno, D. (2011). Cuando las víctimas de violencia escolar se convierten en agresores: “Quién va a defenderme?”. European Journal of Education and Psychology, 3, 177-186.

Estévez, E., Murgui, S., Musitu, G. y Moreno, D. (2008). Adolescent aggression: effects of gender and family and school environments. Journal of Adolescence, 31, 433-450.

Fernández-Ballesteros, R. y Sierra, B. (1989). Escalas de Clima Social FES, WES, CIES y CES. Madrid: TEA.

Galicia, I.X., Sánchez, A. y Robles, F.J. (2009). Factores asociados a la depresión en adolescentes: Rendimiento escolar y dinámica familiar. Anales de Psicología, 25 (2), 227-240.
García, F. y Gracia, E. (2009). Is always authoritative the optimum parenting style? Evidence from Spanish families. Adolescence, 44(173), 101-131.

García, F. y Gracia, E. (2010). What is the optimum parental socialisation style in Spain? A study with children and adolescents aged 10-14 years. Infancia y Aprendiraje, 33, 365-384.

García, J. F., Musitu, G., Riquelme, E. y Riquelme, P. (2011). A confirmatory factor analysis of the "Autoconcepto Forma 5" questionnaire in young adults from Spain and Chile. Spanish Journal of Psychology, 14, 648-658.

Goffin, R. D. (2007). Assessing the adequacy of structural equation models: Golden rules and editorial polices. Personality and Individual Differences, 42, 831-839.

Gouveia-Pereira, M., Vala, J., Palmonari, A. y Rubini, M. (2003). School experience, relational justice and legitimation of institutional. European Journal of Psychology of Education, 18 (3), 309-325.

Hayton, J. C., Allen, D. G. y Scarpello, V. (2004). Factor retention decisions in exploratory factor analysis: A tutorial on parallel analysis. Organizational Research Methods, 7, 191-205.

Izabe, I. y Jaureguizar, J. (2011). ¿Hasta qué punto la violencia filio-parental es bidireccional? Anales de Psicología, 27(2), 265-277.

Little, T. D., Henrich, C. C., Jones, S. M., y Hawley, P. H. (2003). Disentangling the "whys" from the "whats" of aggressive behaviour. International Journal of Behavioral Development, 27, 122-133.

Markland, D. (2007). The golden rule in that there are no golden rules: A commentary on Paul Barrett's recommendations for reporting model fit in structural equation modelling. Personality and Individual Differences, 42, 851-858.

Miles, J. y Shevlin, M. (2007). A time and a place for incremental fit indices. Personality and Individual Differences, 42, 869-874.

Molpeceres, M. A., Llinares, L. y Bernad, J. C. (1999). La percepción de las figuras de autoridad formales e informales y la inclinación a la conducta delictiva en la adolescencia: un análisis preliminar de sus relaciones. Intervención Psicosocial, 8 (3), 349-367.

Moncher, F. J., y Miller, G. E. (1999). Nondelinquent youths' stealing behavior and their perceptions of parents, school, and peers. Adolescence, 34, 577-591.

Moos, R. H. y Trickett, E. J. (1973). Classroom Environment Scale manual. Palo Alto, CA: Consulting Psychologist Press.

Moreno, D., Estévez, E., Murgui, S. y Musitu, G. (2009). Relación entre el clima familiar y el clima escolar: El rol de la empatía, la actitud hacia la autoridad y la conducta violenta en la adolescencia. International Journal of Psychology and Psychological Therapy, 9 (1), 123-136.

Mulaik, S. (2007). There is a place for approximate fit in structural equation modelling. Personality and Individual Differences, 42, 883-891.

Musitu, G. y García, J. F. (2004). Consequences of the family socialization in the Spanish culture. Psicothema, 16, 288-293.

Musitu, G., Estévez, E. y Emler, N. (2007). Adjustment problems in the family and school contexts, attitude towards authority and violent behaviour at school in adolescence. Adolescence, 42, 779-794.

Muthén, B. y Kaplan, D. (1985). A comparison of some methodologies for the factor analysis of non-normal Likert variables. British Journal of Mathematical and Statistical Psychology, 38, 171-189.

Palmonari, A., Rubini, M. y Casoni, E. (1999, July). A psycho-social approach to the study of the orientation toward formal authority. Paper to be presented at the XII General Meeting of the EAESP, Oxford.

Pons, D., Molpeceres, M. A. y Lucas, A. (2000). Experiencia escolar y orientación hacia la autoridad institucional en la adolescencia. Revista de Psicología Social, 15(2), 87-105.

Rigby, K. y Black, D. (1993). Attitudes toward institutional authorities among aboriginal schoolchildren in Australia. Journal of Social Psychology, 133 (6), 845-852.

Rubini, M. y Palmonari, A. (1995). Orientamenti verso la autorita formali e partecipazione poltica degli adolescenti. Giornale Italiano di Psicologia, 10(3), 757-775.

Rubini, M. y Palmonari, A. (1998). The effect of school maladajustment and of membership of adolescent peer-groups on the orientation to the institutional system. Comunicación presentada a la $6^{\text {th }}$ Biennal Conference of the EARA, Budapest. 
Ruiz-Juan, F. y Ruiz-Risueño, J. (2011). Variables predictoras de consumo de alcohol entre adolescentes españoles. Anales de Psicología, 27 (2), 350359 .

Trianes, M. V., Blanca, M. J., de la Morena, L., Infante, L., y Raya, S. (2006). Un cuestionario para evaluar el clima social de la clase y centro. Psicothema, 18 (2), 272-277.
Whorthington, R. L. y Whittaker, T. A. (2006). Scale development research: a content analysis and recommendations for best practices. The Counselling Psychologist, 34 (6), 806-838.

(Artículo recibido: 2-11-2011, revisado: 22-05-2012, aceptado: 28-05-2012) 